\title{
Preparation of PLGA Nanoparticles Loaded with Oxytetracycline Hydrochloride for its Possible Application Against Brucellosis Infection
}

\author{
Rosalva Josefina Rodriguez-Córdova ${ }^{1}$, Karol Yesenia Hernández-Giottonini ${ }^{1}$, Cindy Alejandra \\ Gutiérrez-Valenzuela ${ }^{2}$, Jonathan De la Vega ${ }^{3}$, Reynaldo Esquivel ${ }^{1}$, Paul Zavala-Rivera ${ }^{2}$, Beatriz \\ Arellano-Reynoso $^{3}$, Armando Lucero-Acuña $^{2 *}$ \\ ${ }^{1}$ Nanotechnology Graduate Program, Department of Physics, University of Sonora, Hermosillo, Mexico \\ ${ }^{2}$ Department of Chemical and Metallurgical Engineering, University of Sonora, Hermosillo, Mexico \\ ${ }^{3}$ College of Veterinary Medicine, National Autonomous University of Mexico, Ciudad de Mexico
}

The use of biodegradable polymers for the nanoencapsulation of bioactive compounds represents a promising option for a wide range of applications. Poly-dl-lactic-co-glycolic acid (PLGA) is one of the most successfully used biodegradable polymers in nanomedicine research. One of the reasons of that is because it degrades in the body to biodegradable metabolites; also, practically any hydrophobic or hydrophilic compound could be considered for encapsulation in PLGA nanoparticles (PNP) [1,2]. These PNP could be used as nanocarriers for different compounds in the treatment of brucellosis. Brucellosis is a disease caused by intracellular bacteria of the genus Brucella, a disease which eradication is challenging. Thus, a good strategy to treat this infection is the use of antibiotic nanocarriers which, could prolong the release of them while maintaining a desired concentration on the target site [3]. In the present work, the encapsulation of oxytetracycline hydrochloride (OTC) in PNP (OTC-PNP) was evaluated. The formulation parameters in the encapsulation of OTC by the solvent double-emulsification technique were analyzed.

PNP and OTC-PNP were synthesized following the solvent double-emulsification method [4,5]. Briefly, a specific amount of OTC is dissolved in $0.5 \mathrm{~mL}$ of deionized water and then added to $5 \mathrm{~mL}$ of dichloromethane (DCM) containing $50 \mathrm{mg}$ of PLGA. The mixture was emulsified by sonication for 1 min at $22 \%$ amplitude $(26.5 \mu \mathrm{m})$, then $25 \mathrm{~mL}$ of an aqueous solution of $3 \% \mathrm{w} / \mathrm{v}$ poly(vinyl alcohol) (PVA) were added. The mixture was emulsified again by sonication during $1 \mathrm{~min}$ at $75 \%$ amplitude (90 $\mu \mathrm{m})$ by using a QSonic 500 sonicator under an ice bath. After the emulsification process, the organic solvent was removed by evaporation at room temperature using magnetic stirring at low speed. Finally, nanoparticles were washed using three centrifugation cycles of $20 \mathrm{~min}$ at $37565^{*} \mathrm{~g}$. Particle size, polydispersity index and zeta potential were measured with the Zetasizer Nano ZS. The measures of nanoparticles size were performed by dynamic light scattering (DLS) and the ones for zeta potential by laser Doppler electrophoresis. Size results presented in Fig. 1 not shown significant differences for PNP and OTC-PNP. A low polydispersity index (PDI) for both preparations was found, indicating that the size distribution is homogeneous. Fig. 2 presented zeta potential values around $-20 \mathrm{mV}$ for both preparations, which is related to a fair stability. A summary of these results is presented in Table 1 .

Drug loading (DL) and encapsulation efficiency (EE) were obtained by the determination of OTC concentrations using UV-Vis spectroscopy at $362 \mathrm{~nm}$ with a calibration curve prepared in $10 \mathrm{mM}$ phosphate buffer saline at $\mathrm{pH}$ 7.4. DL is the actual amount of OTC encapsulated per mass of nanoparticles, resulting in $0.33 \pm 0.1 \%$. EE obtained was $3.3 \pm 0.1 \%$ which is described as percent of OTC encapsulated in OTC-PNP with respect to the amount of OTC initially added. Such relatively low encapsulation could be attributed to the diffusion of water-soluble drug in the aqueous phase across the oil phase (DCM) when PLGA nanoparticle are still hardening [6,7]. The surface morphology analysis of 
PNP was obtained by scanning electron microscopy (SEM) through a field emission scanning electron microscope (JSM-7800F), as presented in Figure 3. Samples were prepared by placing a drop of PNP solution on a formvar carbon film and air-dried. Other works where another hydrophilic drugs were encapsulated in PLGA nanoparticles and other materials reports similar results, depending of the particular conditions of the experiments $[4,8,9]$. The system of OTC-PNP has the potential to be used in therapies against intracellular bacteria, such as Brucella.

References:

[1] R. A. Jain, Biomaterials 21 (2000), p. 2475.

[2] F. Danhier et al., J. Control. Release 161 (2012), p. 505.

[3] M. C. Lecaroz et al. Agents Chemother. 51 (2007), p. 1185.

[4] C. A. Gutiérrez-Valenzuela et al., 23 (2017), p. 212.

[5] C. Gutiérrez-Valenzuela et al., Appl. Sci. 6 (2016), p. 364.

[6] H. Kim, B. J. Lee, H. Sah, Drug Deliv. 14 (2007), p. 95.

[7] A. Alshamsan, Saudi Pharm. J. 22 (2014), p. 219.

[8] H. Kim, M. Cho, H. Sah, Arch. Pharm. Res. 28 (2005), p. 370.

[9] F. Serdoz, D. et al Sci. Technol. 18 (2008), p. 404.

[10] The authors acknowledge funding from projects UNISON-USO316003420 and SAGARPA-

CONACYT No 2017-02-291311

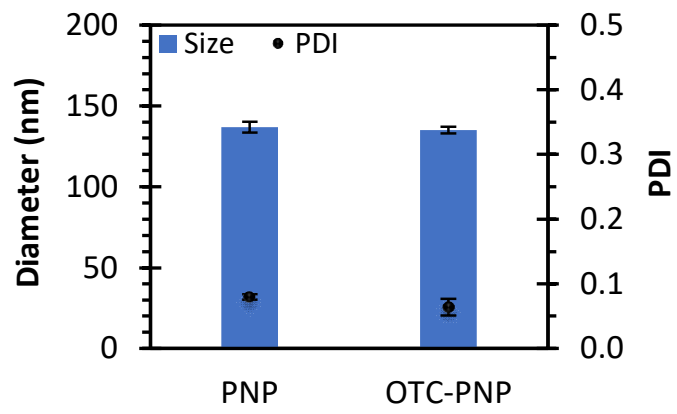

Figure 1. Characteristic of PNP and OTC-PNP

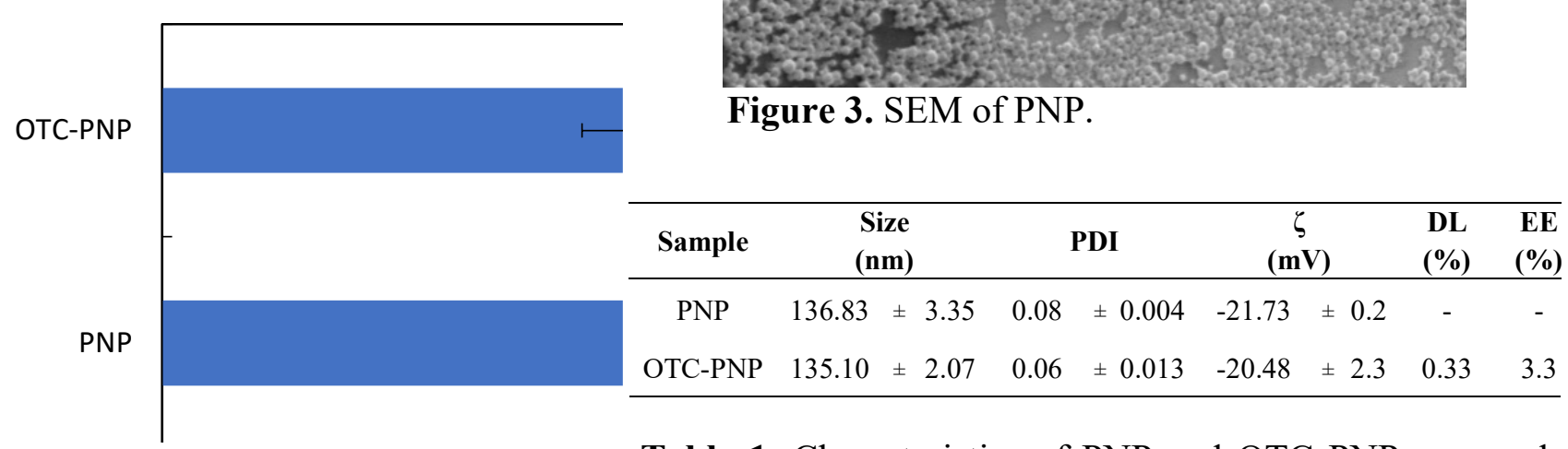

Figure 2. Zeta potential of PNP and OTC-PNP.

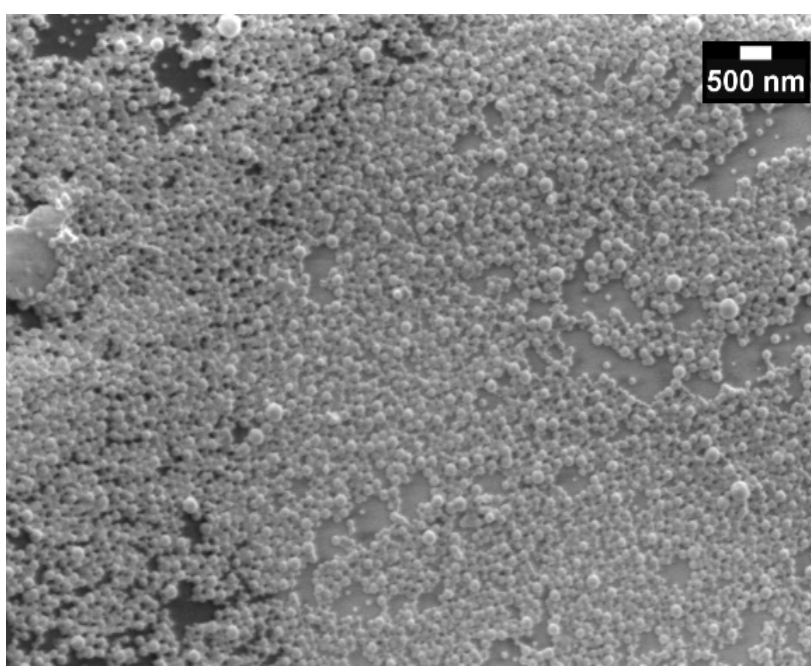

Figure 3. SEM of PNP.

Table 1. Characteristics of PNP and OTC PNP prepared by solvent double-emulsification method. 